\title{
Cutaneous Manifestations of COVID-19: An Experience From Oman
}

Aisha Al Ali ${ }^{1}$, Safiya Al-Shidhani ${ }^{1}$, Fatma Al-Balushi ${ }^{1}$, Mohammed Alhinai ${ }^{2}$, Abdul Rahman Al-Azri ${ }^{3}$, Sultan Al Lawati Al Lawati ${ }^{4}$, Farah Al Ghailani ${ }^{1}$, Reham Al Riyami ${ }^{1}$

1. Dermatology, Al-Nahdha Hospital, Muscat, OMN 2. Internal Medicine, Gastroenterology, Al-Nahdha Hospital, Muscat, OMN 3. Oral Medicine, Al-Nahdha Hospital, Muscat, OMN 4. Internal Medicine, Al-Nahdha Hospital, Muscat, $\mathrm{OMN}$

Corresponding author: Safiya Al-Shidhani, safia_alshidhani@hotmail.com

\section{Abstract}

Objectives: To identify the cutaneous manifestations in COVID-19 disease in Oman.

Methods: The study was conducted in two phases with initial cross-sectional data collection with subsequent telemedical investigations of late skin manifestations including confirmed COVID-19 patients evaluated at Al-Nahdha Hospital and local health centers in Muscat from March 22 to June 2, 2020.

Results: The total number of patients included in the study was 374. Cutaneous manifestations were observed in $1.87 \%(\mathrm{n}=7)$ of patients at presentation with an additional $1.6 \%(\mathrm{n}=5)$ on follow-up. The types of skin reactions included maculopapular rash $(n=6)$, urticaria $(n=2)$, transient pruritic erythema $(n=1)$, pruritic palmoplantar erythema $(n=1)$, pustular eruption $(n=1)$ and flare-up of atopic dermatitis $(n=1)$.

Conclusions: The low percentage of skin lesions is not contradicting previous data and it might just reflect under-reporting of skin rash in the context of the presence of more severe symptoms in our sample population. Skin lesions can still be utilized to treat patients as suspected cases until proven otherwise as it can be a silent clue in asymptomatic patients.

Review began 06/12/2021 Review ended 07/26/2021 Published 07/27/2021

\section{() Copyright 2021}

Al Ali et al. This is an open access article distributed under the terms of the Creative Commons Attribution License CC-BY 4.0., which permits unrestricted use, distribution, and reproduction in any medium, provided the original author and source are credited.
Categories: Dermatology, Infectious Disease

Keywords: cutaneous manifestations, sars-cov-2, corona virus, skin manifestations, covid-19

\section{Introduction}

Since late December 2019, the COVID-19 outbreak has spread from Wuhan, China to the rest of the world causing significant impacts on health systems and the global economy. COVID-19 infection is now well described in terms of its presentation and disease course. The incubation period of COVID-19 is estimated to be around five days [1]. It generally produces non-specific symptoms like body aches, fever and cough that might be associated with a variety of other symptoms like sore throat, shortness of breath, chest pain, nausea, diarrhea and headache among other less frequent symptoms [1,2].

One of the initial studies in Italy reported just above $20 \%$ of COVID-19 patients developing skin lesions. The reported skin lesions included erythematous rash, vesicles and generalized urticaria. No specific association with disease stage or severity was reported by the same investigators [3]. Large-scale studies and case reports were subsequently released reporting more extensive illustrations and classification of skin associations that were seen in confirmed or suspected COVID-19 patients with skin rash [4].

It was assumed that these lesions could be induced directly by the virus effect or secondary to associated complications resulting in vascular inflammation and occlusion caused by coagulation abnormalities and vascular damage due to COVID-19 [5-7].

There is limited data on the cutaneous manifestations of COVID-19 and the experiences in the middle east countries in general and in Oman in particular, therefore we report here the outcome of a cross-sectional study that focused on the cutaneous lesions seen in patients who were affected by COVID-19 within two weeks after confirmation of disease.

\section{Materials And Methods}

The study was conducted in two phases. The first phase included cross-sectional analysis of COVID-19 patient demographics and clinical presentation with a focus on skin manifestation at the time of diagnosis, followed by a second phase of telemedical investigation of any subsequent late skin presentation.

The sample size for the study was calculated with a $95 \%$ confidence level based on the population of all confirmed patients with COVID-19 in Oman up to May 5, 2020. The estimated sample size needed was 373 


\section{Cureus}

subjects and the actual sample that was included in the study is 374. Data was extracted from Al Shifa Health Care computer system and recorded electronically using an Excel sheet. Data were analyzed and reported descriptively.

At the first phase, all COVID-19 patients who presented from March 22 to June 2, 2020, as outpatients or admitted as inpatients at Al-Nahdha Hospital, and all outpatients from COVID-19 focal health centers in Muscat Governorate, were included in the study. Patients were included if they had positive severe acute respiratory syndrome coronavirus 2 (SARS-CoV-2) RNA polymerase chain reaction (PCR) test who presented in the period between March 22 to June 2, 2020, and were residents in Muscat Governorate. Patients who had been diagnosed with COVID-19 outside Muscat Governorate were excluded. The data collected included basic demographics, medical background and COVID-19 examination details (Table 1).

\begin{tabular}{|c|c|c|c|c|}
\hline & Male & Female & Subtotal & Grand total \\
\hline \multicolumn{5}{|l|}{ Age Group } \\
\hline 0-19 & $8(2 \%)$ & $11(3 \%)$ & 19 & \multirow{5}{*}{374} \\
\hline $20-39$ & $109(29 \%)$ & $34(9 \%)$ & 143 & \\
\hline $40-59$ & $119(32 \%)$ & $34(9 \%)$ & 153 & \\
\hline 60+ & $38(10 \%)$ & $21(6 \%)$ & 59 & \\
\hline \multicolumn{4}{|l|}{ Health institution } & \\
\hline Al Nahdha Hospital & $251(67.2 \%)$ & $77(20.4 \%)$ & 328 & \multirow{2}{*}{374} \\
\hline Health center & $23(6.2 \%)$ & $23(6.2 \%)$ & 46 & \\
\hline \multicolumn{5}{|l|}{ Nationality } \\
\hline Non-Omani & $179(47.8 \%)$ & $28(7.5 \%)$ & 207 & \multirow{3}{*}{374} \\
\hline Omani & $95(25.5 \%)$ & $72(19.2 \%)$ & 167 & \\
\hline \multicolumn{4}{|l|}{ Associated comorbidities } & \\
\hline Hypertension & $59(16 \%)$ & $27(7 \%)$ & 86 & \multirow{6}{*}{374} \\
\hline Diabetes mellitus & $72(19.2 \%)$ & $23(6.2 \%)$ & 95 & \\
\hline Asthma & $13(3.5 \%)$ & $13(3.5 \%)$ & 26 & \\
\hline Coronary artery disease & $14(3.7 \%)$ & $1(0.3 \%)$ & 15 & \\
\hline Hyperlipidemia & $17(4.6 \%)$ & $5(1.3 \%)$ & 22 & \\
\hline Kidney disease & $6(1.6 \%)$ & $4(1.1 \%)$ & 10 & \\
\hline
\end{tabular}

TABLE 1: Demographic \& clinical characteristics of COVID-19 patients in Muscat

In the second phase of the study, the same cohort of patients included initially were further contacted with a specific set of questions to investigate the development of any late skin presentation. A total of 310 out of the initial cohort were followed up, after exclusion of confirmed dead patients, unreachable patients and those who had skin lesions at initial presentation. A telemedicine clinic was set up for screening patients remotely. Patients were informed about the purpose of the telemedical encounter and consent was taken verbally. Briefly, history was taken regarding the development of skin lesions within the following two weeks of confirmed diagnosis of COVID-19 disease. Patients were asked to describe the location, associated symptoms, exposure to any medications and the duration until resolution of the lesions. Furthermore, a pictorial reference was provided to the participants who confirmed the development of skin lesions for a better description. All data were added to the original datasheet of the first phase.

\section{Results}

A total of 374 patients confirmed with COVID-19 disease by using SARS-CoV-2 RNA PCR were enrolled in the study. Fifty-two percent ( $\mathrm{n}=195,52 \%)$ were admitted as inpatients at Al-Nahdha Hospital, 13\% $(\mathrm{n}=48)$ were intubated and transferred to Intensive Care Units at other tertiary hospitals and 35\% ( $n=131)$ were managed as outpatients with home-based care and quarantine. The majority of cases included in the study attended Al Nahdha Hospital $(n=328,88 \%)$, whereas $12 \%(n=46)$ were evaluated at local health centers and 


\section{Cureus}

managed with home-based care and quarantine. Omani nationals accounted for $45 \%(\mathrm{n}=167)$ and nonOmani patients accounted for $55 \%(\mathrm{n}=207)$.

The mean age of affected patients was 43.5 years ( $\mathrm{SD}=15.27$, age range: 25 days- 85 years). There were more males than females [73.3\% ( $n=274)$ males, 26.7\% ( $n=100)$ females]. The most affected age group was between 30 and 49 years old with a total of $50.5 \%(n=189)$. A total of $248(66.2 \%)$ of patients were younger than 50 years old and $33.7 \%(n=126)$ were 50 years old and above. The demographic and pre-existing medical conditions of the sample collected are demonstrated in Table 1 . The incidence of clinical features developed following COVID-19 infection in these patients is shown in Figure 1.

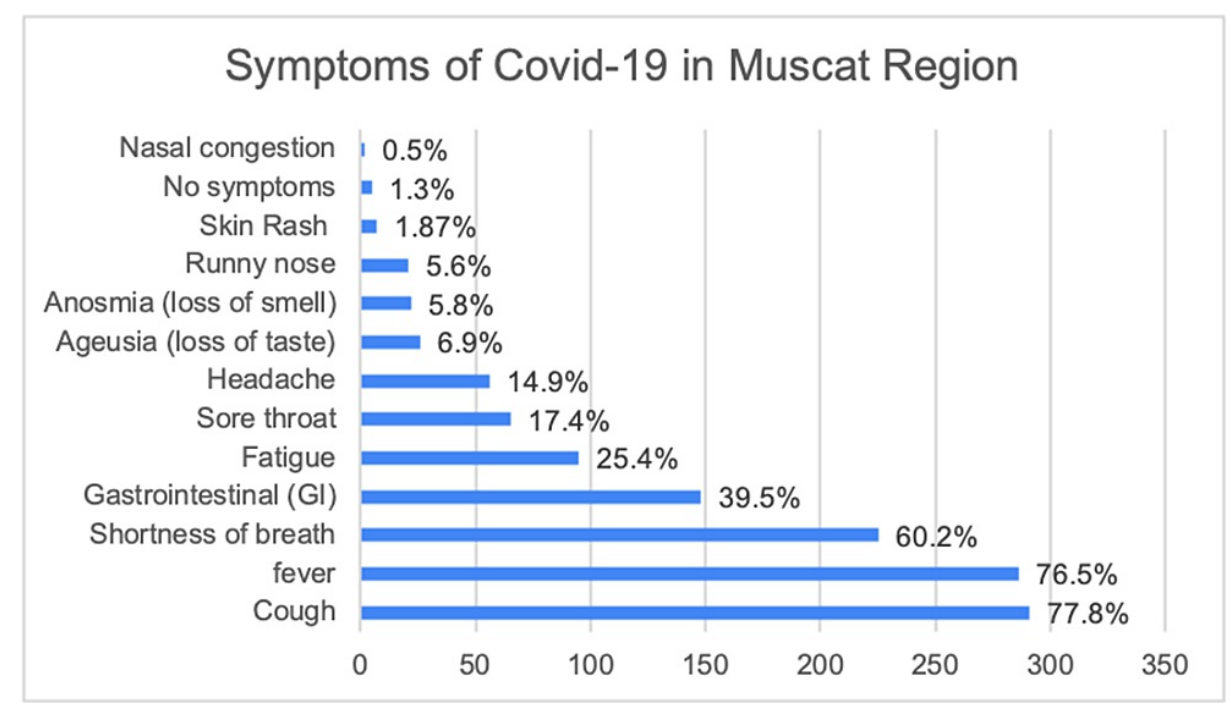

FIGURE 1: Symptoms of COVID-19 in Muscat Region

Skin lesions were documented in $1.87 \%(\mathrm{n}=7)$ of the 374 patients. The types of skin lesions included maculopapular rash $(n=2)$ (Figure 2), transient pruritic erythema $(n=1)$ (Figure 3), urticaria $(n=1)$ (Figure 4), palmoplantar erythema with itching $(\mathrm{n}=1)$ and pustular eruption with erythematous background $(\mathrm{n}=1)$ (Figure 5).One patient had a flare-up of pre-existing atopic dermatitis.

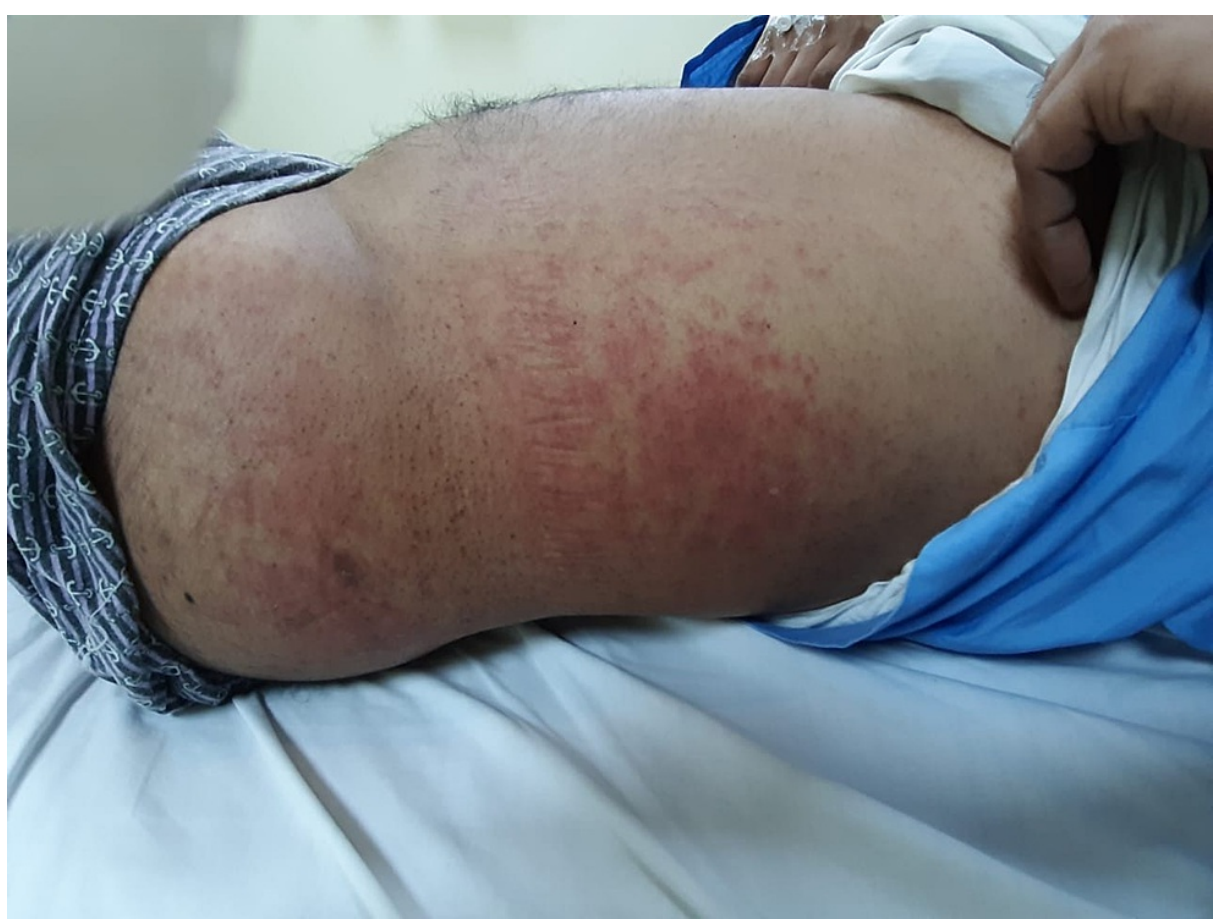

FIGURE 2: maculo-papular rash 


\section{Cureus}

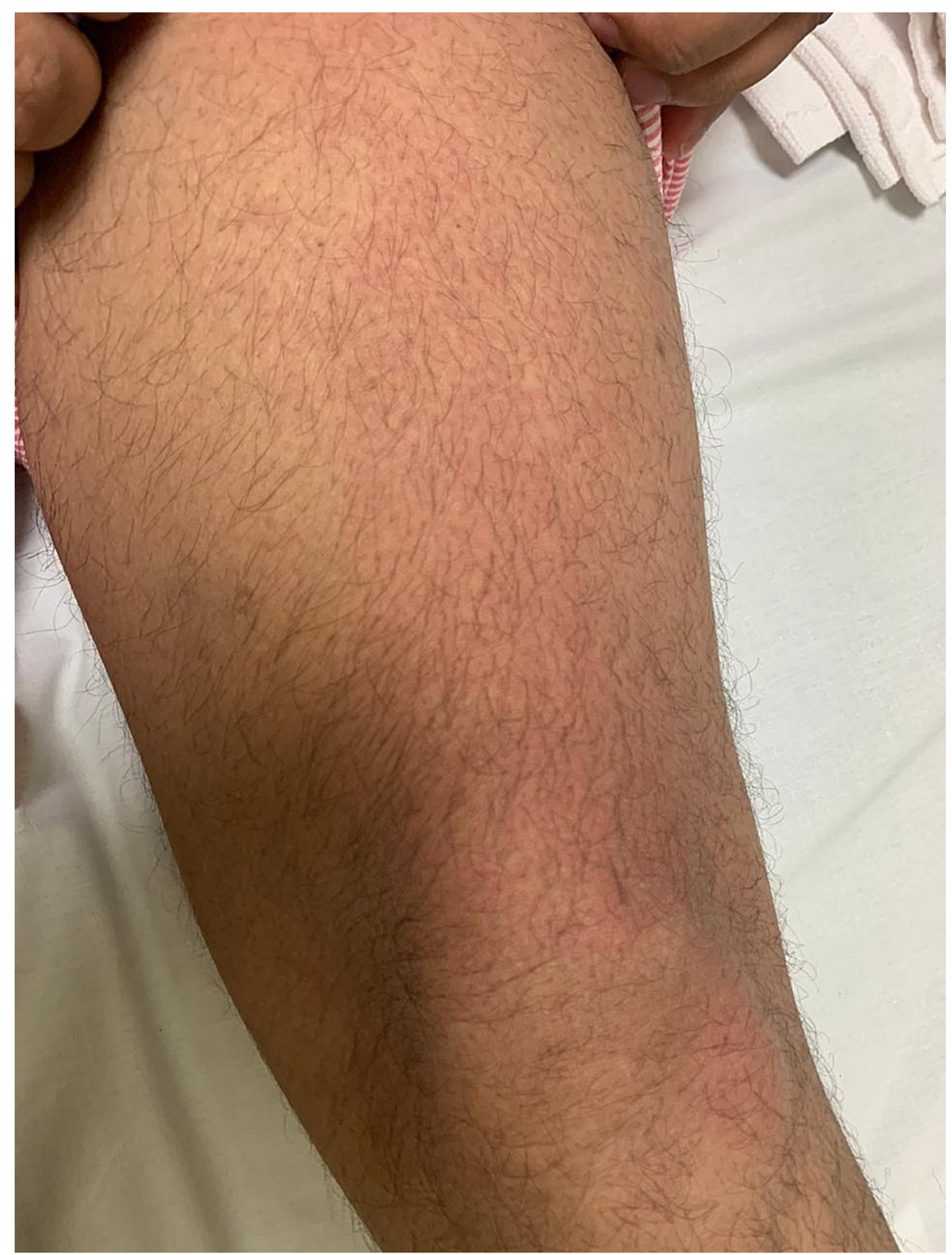

FIGURE 3: transient pruritic erythema 


\section{Cureus}

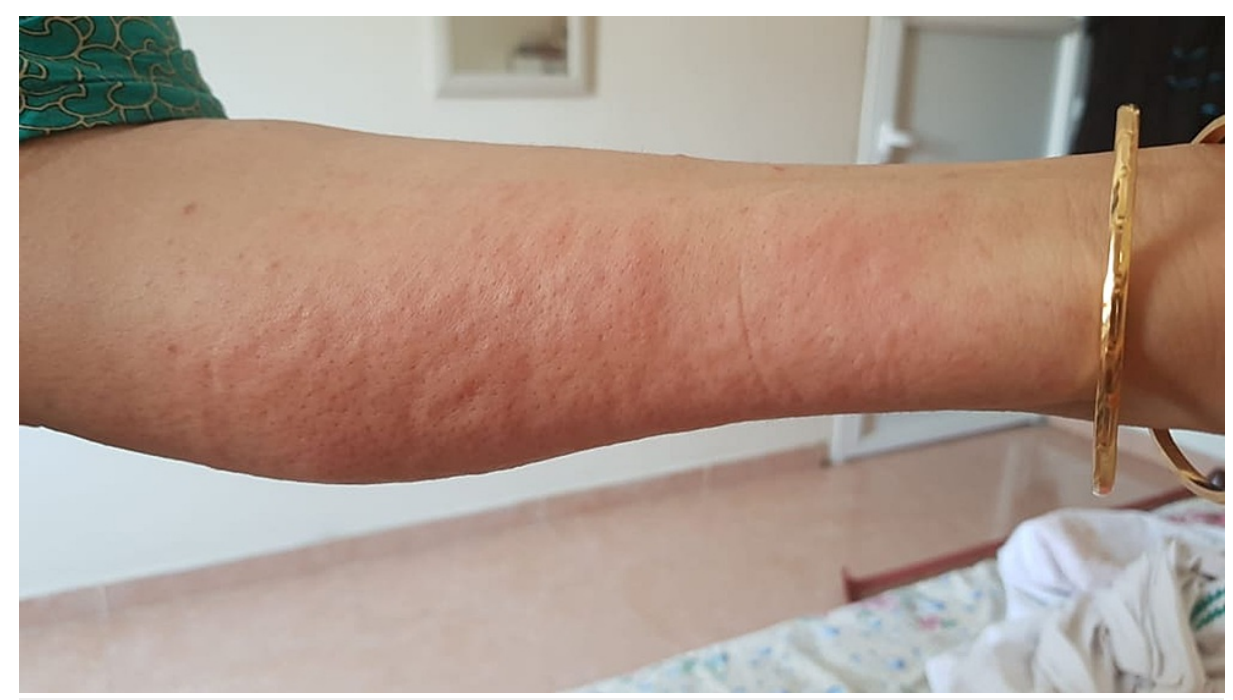

FIGURE 4: Urticarial Eruption

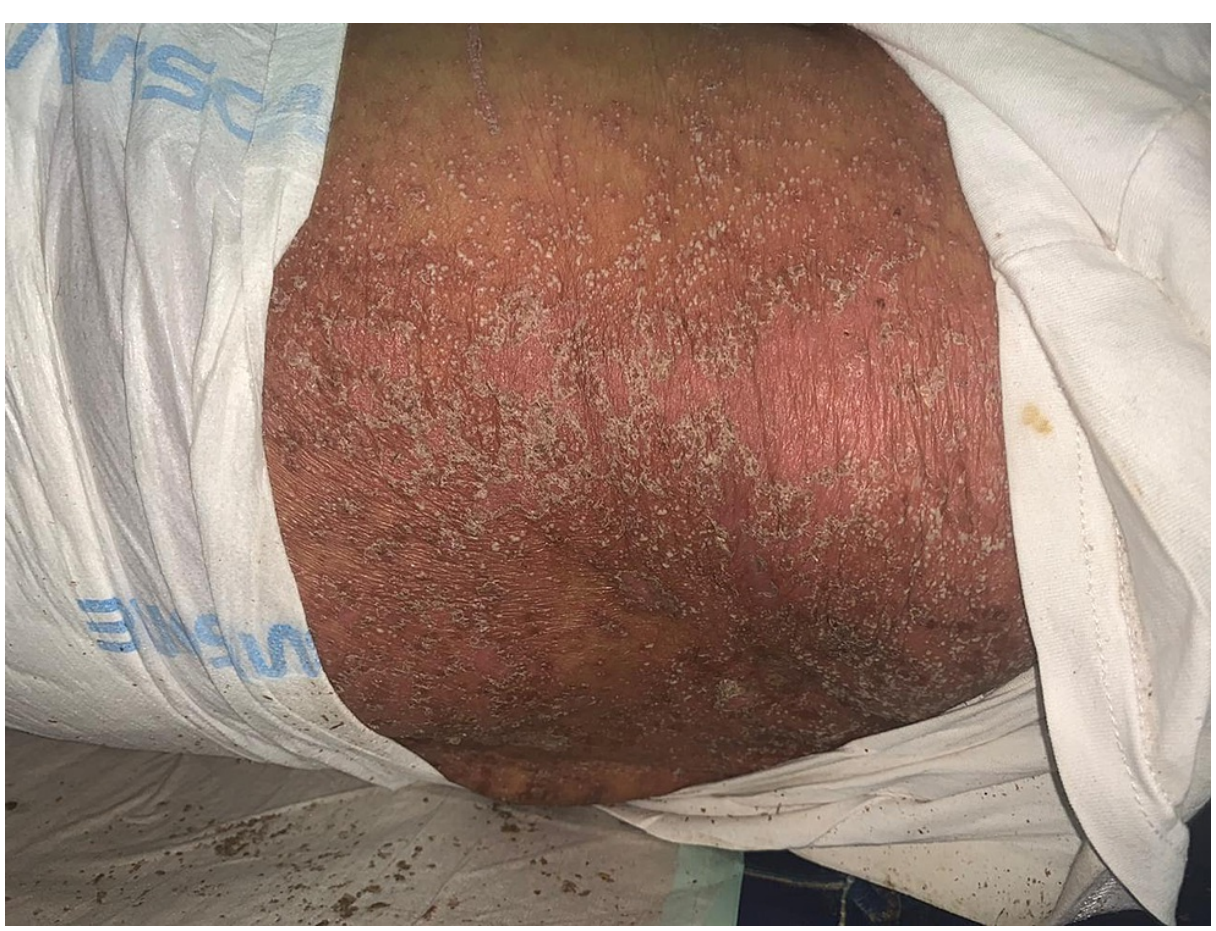

FIGURE 5: pustular eruption with erythematous background

The subsequent telemedical follow-up revealed an additional five patients (out of 310 reachable patients) who developed skin rash (1.6\%) within two weeks of confirming COVID-19 infection. Four of them had maculopapular rash and one urticarial rash. Skin lesions healed without using topical treatments except in one patient with maculopapular rash. There was preceding history of different drug intake with combinations of antibiotics, hydroxychloroquine and antivirals before the onset of the rash in all of the five patients.

\section{Discussion}

In this study, we described the skin manifestations in COVID-19 inpatients and outpatients who have been evaluated at Al Nahdha Hospital and local health centers in Muscat Governorate in Oman.

The majority of affected patients were middle-aged men. The mean age of included patients was 43.5 years $(\mathrm{SD}=15.27)$, which is comparable with what was reported in an international registry of 716 patients from 31 countries [8]. However, the mean age in our study was younger than some previous large reported studies 
$[2,9]$. There was an obvious male predominance which is consistent with other published international studies $[9,10]$.

During the time of the study, it was noticed that non-nationals were more commonly affected compared to the Omani population although we included all patients with COVID-19 who were seen during the period from March 22 to June 2 regardless of their nationalities. Several factors have been hypothesized to explain this observation including the ratio of national to non-nationals in the Muscat Governorate region, possibly less optimal lifestyle as some are living in groups, crowded rooms and campuses with possible financial barriers [11].

In our study, the associated chronic medical conditions were seen less frequently than other reported studies $[2,9]$. Diabetes mellitus was the most common co-morbidity followed by hypertension, asthma and dyslipidemia. Most of the patients had cough and fever as presenting symptoms followed by shortness of breath which is consistent with previous reports [11-13].

Skin lesions were seen in $1.87 \%$ at initial presentation of all affected patients and an additional $1.6 \%$ developed skin lesions within the following two weeks. This is smaller than most of the reported studies, with the highest reported incidence of $20.4 \%$ of all COVID-19 patients from the first prospective study in Italy [3]. Although, a very low incidence of only $0.2 \%$ was reported in one of the initial Chinese studies of 1099 positive cases [14]. In the first phase of the study, skin lesions presented variably as maculopapular rash, transient pruritic erythema, urticaria, palmoplantar erythema with itching, pustular eruption with erythematous background and flare-up of pre-existing atopic dermatitis. Patients who developed skin lesions had them mainly along with other symptoms of COVID-19 before the initiation of any treatment and these included the maculopapular eruption, transient pruritic erythema, palmoplantar pruritic erythema and flare-up of pre-existing atopic dermatitis. Some patients developed pustular eruption with erythematous background and urticarial rash that presented after initiation of treatment.

In our study, the additional five patients who noticed skin lesions within two weeks of infection had a preceding history of drug intake before the onset of the rash. Four of them had maculopapular eruption and one had urticarial wheels. The total accumulative number of patients with skin lesions from our study was 12. The number of patients who developed different skin lesions is illustrated in Table 2.

\begin{tabular}{|l|c|}
\hline Type of lesion & Number of patients \\
\hline atopic dermatitis flare-up & 1 \\
\hline erythematous rash (maculopapular rash) & 6 \\
palmoplantar erythema with itching & 1 \\
pustular eruption with erythema (AGEP) drug eruption & 1 \\
transient pruritic erythematous patch on thighs, lasted 48 hours & 1 \\
urticaria & 2 \\
Total & 12 \\
\hline
\end{tabular}

\section{TABLE 2: Types of skin lesions reported by COVID-19 patients}

The maculopapular rash was the most common skin lesion observed in confirmed COVID-19 cases followed by urticaria in our study, and this is consistent with what was reported in the literature $[3,4,8]$. These two skin eruptions are non-specific findings and can present with many other viral infections or as an adverse reaction to drugs. Since these symptoms are not specific, they might not be helpful for early diagnosis of COVID-19 disease particularly. They may, however, raise the suspicion of any viral illness including COVID19 infection.

The transient pruritic erythema on extremities and trunk and the palmoplantar pruritic erythema were noticed at the same time as other symptoms and resolved with symptomatic treatment. These manifestations might be more potentially associated with COVID-19 disease but there was no clear reported data with similar presentations until recently, where palmoplantar involvement was frequently found and reported [15]. One patient had a severe flare-up of pre-existing atopic dermatitis that required high doses of systemic steroids.

The last case of skin reaction with pustular eruption is more likely to be a drug reaction, known as acute generalized exanthematous pustulosis (AGEP), triggered by ceftriaxone that was part of the patient's 
regimen treatment. The diagnosis of AGEP was clinical and the lesions resolved gradually after discontinuation of this medication along with symptomatic treatment. The improvement of the patient's condition after stopping the offending drug supported the diagnosis of AGEP. The prescription of multiple drugs for COVID-19 patients increases the likelihood of developing adverse drug reactions and this should be considered as a possible cause for developing skin lesions.

Many other skin lesions have been reported in other studies but were not seen in our sample population. In one study, among skin lesions reported in confirmed and suspected cases of COVID-19, acral lesions were unexpectedly common, presenting in $51 \%$ of patients with chilblain-like lesions being the most frequent presentation [16]. This was also reported in a recent study where a high percentage of chilblain-like acral pattern was observed in $24.6 \%$ of the population included in the study [17].

It was explained in several studies that chilblain-like lesions could be linked with COVID-19 given that these lesions were observed during warm weather which is against the usual natural history for development of chilblain, increasing incidence observed by dermatologists during that period and the presence of history of contact with COVID-19 cases. However, in most cases, chilblain was noticed later in the disease course which was hypothesized to be related to the microthrombi which is expected due to the deranged coagulation profile noticed in severe COVID-19 patients $[4,16,18]$. It has been observed in a recent study that pernio/chilblain were recurrent in the sample population with subsequent cold exposure [19].

Some of the skin manifestations reported in other studies suggest the possibility of vascular injury, inflammation and thrombotic vasculopathy as potential underlying pathophysiology. Those manifested as petechial rash, Kawasaki disease in pediatric and adult patients, livedo reticularis, livedo racemose, acral ischemia and retiform purpura [5-7,20-23]. In addition, pityriasis rosea-like lesions were reported as an associated skin rash in confirmed cases of COVID-19 disease [24]. These less common presentations were not encountered in our study. This might be attributed to different factors, including different genetic background, younger population with fewer co-morbidities and different genomes of SARS-CoV-2. Furthermore, it can be cautiously suggested that skin lesions might be underreported by patients and physicians as vigilance to skin lesions is lower compared to the other more serious symptoms of COVID-19.

\section{Conclusions}

We describe the incidence of skin involvement in patients who have contracted COVID-19 infection in Muscat Governorate in Oman. Skin manifestation of COVID-19 is not among the most commonly reported clinical presentation that alarm physicians to suspect the disease. We showed a low incidence of skin involvement in our sample compared to other reported incidence. However, at the time of the pandemic, unexplained skin lesions with or without other respiratory symptoms should alert physicians to consider COVID-19 as a cause. This will help minimize the spread of the disease. To our knowledge, this is the first report describing the skin manifestations of COVID-19-infected patients in Oman. More data that can represent the experience of other countries in the region and the pattern of skin involvement are needed.

\section{Additional Information \\ Disclosures}

Human subjects: Consent was obtained or waived by all participants in this study. Regional Research and Ethics committee in Muscat Governorate. issued approval MH/DGHS/DPT/593/2020. The ethical approval for the study was granted from Ministry of Health, Regional Research and Ethics committee in Muscat Governorate. . Animal subjects: All authors have confirmed that this study did not involve animal subjects or tissue. Conflicts of interest: In compliance with the ICMJE uniform disclosure form, all authors declare the following: Payment/services info: All authors have declared that no financial support was received from any organization for the submitted work. Financial relationships: All authors have declared that they have no financial relationships at present or within the previous three years with any organizations that might have an interest in the submitted work. Other relationships: All authors have declared that there are no other relationships or activities that could appear to have influenced the submitted work.

\section{Acknowledgements}

Dr. Manar Alsana Ali ALZeedi, Family Physician, Ruwi Health Center. Dr. Amira Salim ALAdi, Family Physician, AL Nahdha Health Center. Mr. Nasser Hamed AL Saadi, Assistant Supervisor of health information management (medical statistician), AL Nahdha Hospital.

\section{References}

1. Wu YC, Chen CS, Chan YJ: The outbreak of COVID-19: an overview. J Chin Med Assoc. 2020, 83:217-20. 10.1097/JCMA.0000000000000270

2. Wang D, Hu B, Hu C, et al.: Clinical characteristics of 138 hospitalized patients with 2019 novel coronavirus-infected pneumonia in Wuhan, China. Jama. 2020, 17:1061-9. 10.1001/jama.2020.1585

3. Recalcati S: Cutaneous manifestations in COVID-19: a first perspective. J Eur Acad Dermatol Venereol. 2020, 34:e212-3. 10.1111/jdv.16387 
4. Galván Casas C, Català A, Carretero Hernández G, et al.: Classification of the cutaneous manifestations of COVID-19: a rapid prospective nationwide consensus study in Spain with 375 cases. Br J Dermatol. 2020, 183:71-7. 10.1111/bjd.19163

5. Zhang Y, Cao W, Xiao M, et al.: Clinical and coagulation characteristics in 7 patients with critical COVID2019 pneumonia and acro-ischemia. Zhonghua Xue Ye Xue Za Zhi. 2020, 41:302-7. 10.3760/cma.j.issn.02532727.2020.008

6. Arachchillage DR, Laffan M: Abnormal coagulation parameters are associated with poor prognosis in patients with novel coronavirus pneumonia. J Thromb Haemost. 2020, 18:1233-4. 10.1111/jth.14820

7. Shaigany S, Gnirke M, Guttmann A, et al.: An adult with Kawasaki-like multisystem inflammatory syndrome associated with COVID-19. Lancet. 2020, 396:e8-e10. 10.1016/S0140-6736(20)31526-9

8. Freeman EE, McMahon DE, Lipoff JB, et al.: The spectrum of COVID-19-associated dermatologic manifestations: an international registry of 716 patients from 31 countries. J Am Acad Dermatol. 2020, 83:1118-29. 10.1016/j.jaad.2020.06.1016

9. Grasselli G, Zangrillo A, Zanella A, et al.: Baseline characteristics and outcomes of 1591 patients infected with SARS-CoV-2 admitted to ICUs of the Lombardy Region, Italy. JAMA. 2020, 323:1574-81. 10.1001/jama.2020.5394

10. Wang R, Pan M, Zhang X, et al.: Epidemiological and clinical features of 125 Hospitalized Patients with COVID-19 in Fuyang, Anhui, China. Int J Infect Dis. 2020, 95:421-8. 10.1016/j.ijid.2020.03.070

11. Khamis F, Al-Zakwani I, Al Naamani H, et al.: Clinical characteristics and outcomes of the first 63 adult patients hospitalized with COVID-19: An experience from Oman. J Infect Public Health. 2020, 13:906-13. Accessed: Jun 8: 10.1016/j.jiph.2020.06.002

12. Chen N, Zhou M, Dong X, et al.: Epidemiological and clinical characteristics of 99 cases of 2019 novel coronavirus pneumonia in Wuhan, China: a descriptive study. Lancet. 2020, 395:507-13. 10.1016/S01406736(20)30211-7

13. Rodriguez-Morales AJ, Cardona-Ospina JA, Gutiérrez-Ocampo E, et al.: Clinical, laboratory and imaging features of COVID-19: a systematic review and meta-analysis. Travel Med Infect Dis. 2020, 34:101623. 10.1016/j.tmaid.2020.101623

14. Guan WJ, Ni ZY, Hu Y, et al.: Clinical characteristics of coronavirus disease 2019 in China . N Engl J Med. 2020, 382:1708-20. 10.1056/NEJMoa2002032

15. Nuno-Gonzalez A, Martin-Carrillo P, Magaletsky K, et al.: Prevalence of mucocutaneous manifestations in 666 patients with COVID-19 in a field hospital in Spain: oral and palmoplantar findings. Br J Dermatol. 2021, 184:184-5. 10.1111/bjd.19564

16. de Masson A, Bouaziz JD, Sulimovic L, et al.: Chilblains is a common cutaneous finding during the COVID19 pandemic: a retrospective nationwide study from France. J Am Acad Dermatol. 2020, 83:667-70. 10.1016/j.jaad.2020.04.161

17. Marzano AV, Genovese G, Moltrasio C, et al.: The clinical spectrum of COVID-19-associated cutaneous manifestations: an Italian multicenter study of 200 adult patients. J Am Acad Dermatol. 2021, 84:1356-63. 10.1016/j.jaad.2021.01.023

18. Mazzotta F, Troccoli T: Acute acro-ischemia in the child at the time of COVID-19. Eur J Pediatr Dermatol. 2020, 8:71-4. 10.26326/2281-9649.30.2.2102

19. Freeman EE, McMahon DE, Lipoff JB, et al.: Cold and COVID: recurrent pernio during the COVID-19 pandemic. Br J Dermatol. 2021, 185:214-6. 10.1111/bjd.19894

20. Joob B, Wiwanitkit V: COVID-19 can present with a rash and be mistaken for Dengue . J Am Acad Dermatol. 2020, 82:e177. 10.1016/j.jaad.2020.03.036

21. Jimenez-Cauhe J, Ortega-Quijano D, Prieto-Barrios M, Moreno-Arrones OM, Fernandez-Nieto D: Reply to "COVID-19 can present with a rash and be mistaken for dengue": Petechial rash in a patient with COVID-19 infection. J Am Acad Dermatol. 2020, 83:e141-2. 10.1016/j.jaad.2020.04.016

22. Jones VG, Mills M, Suarez D, et al.: COVID-19 and Kawasaki disease: novel virus and novel case . Hosp Pediatr. 2020, 10:537-40. 10.1542/hpeds.2020-0123

23. Llamas-Velasco M, Muñoz-Hernández P, Lázaro-González J, Reolid-Pérez A, Abad-Santamaría B, Fraga J, Daudén-Tello E: Thrombotic occlusive vasculopathy in a skin biopsy from a livedoid lesion of a patient with COVID-19. Br J Dermatol. 2020, 183:591-3. Accessed: May 14: 10.1111/bjd.19222

24. Johansen M, Chisolm SS, Aspey LD, Brahmbhatt M: Pityriasis rosea in otherwise asymptomatic confirmed COVID-19-positive patients: a report of 2 cases. JAAD Case Rep. 2021, 7:93-4. 10.1016/j.jdcr.2020.10.035 